\title{
Stabilization of a hyperbolic PDEs-ODE network using a recursive dynamics interconnection framework
}

\author{
Jean Auriol $^{1}$, Federico Bribiesca-Argomedo ${ }^{2}$, Silviu-Iulian Niculescu ${ }^{1}$ and Jeanne Redaud ${ }^{1}$
}

\begin{abstract}
In this paper, we design a state-feedback law that exponentially stabilizes an underactuated network of scalar hyperbolic systems coupled at the end of the chain with a finite-dimensional system modeled by an ordinary differential equation. Our approach uses a recursive dynamics interconnection framework. More precisely, for each subsystem, we solve a stabilization problem, a tracking problem and design a statepredictor. Then, we are able to recursively design a control law that stabilizes the whole chain. Some illustrative examples complete the presentation.
\end{abstract}

\section{INTRODUCTION}

Interconnected systems represented by Partial Differential Equations (PDEs) naturally appear when modeling industrial processes. Some well-known examples include traffic network systems with different types of vehicles [15], [46], ventilation in buildings [35], density-flow systems [10], [25], open channels [22], [23], [24], communication networks [19]. Additional couplings with Ordinary Differential Equations (ODEs) arise when there is a lumped element coupled to the distributed dynamics such as in heavy chain systems [34], Rijke tubes [17] (where the ODE is sandwiched between two PDEs) or mechanical vibrations in drilling applications [36]. In the last case case, the hyperbolic PDE system models axial and torsional stress propagation (with potential discontinuities due to the junctions between different types of pipes), while the ODE represents the dynamics of the Bottom hole Assembly (BHA). Several approaches have been proposed in the literature to stabilize such interconnected systems. Among them, we can cite PI boundary controllers in the case of fully actuated networks [10], flatness-based designs [37], or the method of characteristics for quasilinear hyperbolic systems in a treelike networks [23], [44]. Recently, this last approach has been used in [39] to design output-feedback laws for networks of interconnected scalar semilinear PDEs. However, the proposed state-feedback control law requires solving a set of PDEs online which is computationally expensive. Regarding networks of ODEs and PDEs, the backstepping approach [28] has been shown to be a powerful tool for the design of explicit stabilizing control laws. It was first applied to hyperbolic equations in [28] to express the delays acting on an ODE as PDE states of transport equations. This led to a new interpretation of the delay-compensating Finite Spectrum Assignment controller. Based on extensions of the backstepping technique, more complex stabilization problems have been progressively solved: non-linear ODEs with delays [12], ODEs coupled with a beam [45], or velocity

\footnotetext{
${ }^{1}$ Jean Auriol, Silviu-Iulian Niculescu and Jeanne Redaud are with Université Paris-Saclay, CNRS, Inria, CentraleSupélec, Laboratoire des Signaux et Systèmes, 91190, Gif-sur-Yvette, France. Corresponding author: jean.aurioldcentralesupelec.fr ${ }^{2}$ Federico Bribiesca-Argomedo is with Univ Lyon, INSA Lyon, CNRS, Ampère, F69621, Villeurbanne, France.
}

recirculation in a wave equation [40]. The backstepping approach has been successfully used in [5], to stabilize a system of hyperbolic PDEs coupled with an ODE in a PDE-ODE structure. In the case of a scalar PDE system, [27] solved a similar problem (with a potentially non-linear ODE) by solving a tracking problem. The ODE-PDE-ODE structure has been considered in [43], [18], [20] and recently in [13] with the design of a strictly proper control design. In recent contributions [2], the authors have stabilized systems with a PDE-ODE-PDE structure. Finally, the general problem of the stabilization of an arbitrary number of interconnected scalar PDEs has been solved in [4], introducing a complex backstepping transformation.

So far, one major limitation of the backstepping approach for the stabilization of general networks of interconnected ODE-PDE systems is its lack of genericity, i.e., the involved backstepping transformations are specific to the system under consideration. More precisely, adding a new subsystem into the network implies designing a completely new backstepping transformation to encompass this new subsystem.

In other research fields, such as electronics [3] or bioengineering [38], [41], as well as in the process industry [29], modular approaches have been developed in order to efficiently model complex interconnected systems.

In this paper, we develop a new recursive interconnected dynamics framework to design an explicit state-feedback control law that stabilizes a network of interconnected scalar linear hyperbolic systems coupled with an ODE at the end of the chain. Roughly speaking, the proposed control law is recursively obtained by considering stabilizing virtual inputs for each subsystems and by ensuring the outputs of each subsystem converge to these desired virtual inputs. The control design becomes simpler and is based on simple assumptions that can be independently verified for each subsystem. This new framework allows for a "plug-and-play"-like approach to control design since additional subsystems, satisfying similar conditions, can be added to the network using the same procedure.

The approach followed in this paper consists on solving three independent problems for each subsystem: (i) a stabilization problem, (ii) a delayed-trajectory tracking problem and (iii) building a predictor for the system. If we consider the specific case of the last subsystem (the one furthest in the chain from the control action), it means in particular that we know an input that stabilizes it. Then we are able, using the second-to last subsystem, to track this desired signal (with inevitable delay due to finite speeds of propagation, which is compensated using the predictor of the last subsystem). Iterating this procedure, at each step we know a control input that solves the stabilization problem for all downstream subsystems and we use the current subsystem to track this trajectory, compensating the inevitable delay using the predictor for the downstream subsystem, which is 
constructed using a composition of the subsystem predictors.

This paper is structured as follows: in Section II, the control problem is presented, as well as the general ideas behind our new recursive dynamics interconnection framework. In Section III, we present necessary conditions to apply this framework and show that for the class of systems under consideration, they are always satisfied. We design the stabilizing control law in Section IV and present some simulation results in Section V.

\section{PROBLEM DESCRIPTION}

\section{A. System under consideration}

We consider in this paper a system composed of a chain of $n>1$ subsystems interconnected through their boundaries. Each subsystem consists of two coupled linear hyperbolic PDEs, except for the last one that can be an ODE. This class of system may appear when considering oil production systems made of networks of pipes (whose principal line is known as the manifold) [33]. More precisely, the lower part of the drill-string is usually made up of drill collars that can have a great impact on the global dynamics due to their inertia [1]. In particular, these pipes may have different lengths, density, inertia or Young's modulus. These spatial variations in the characteristic line impedance may cause reflections to appear in the junctions. Each subsystem (except for the last one) is defined by the following set of PDEs $(i \in\{1, \cdots, n-1\})$

$$
\begin{aligned}
& \partial_{t} u_{i}(t, x)+\lambda_{i} \partial_{x} u_{i}(t, x)=\sigma_{i}^{+}(x) v_{i}(t, x), \\
& \partial_{t} v_{i}(t, x)-\mu_{i} \partial_{x} v_{i}(t, x)=\sigma_{i}^{-}(x) u_{i}(t, x),
\end{aligned}
$$

evolving in $\{(t, x)$ s.t. $t>0, x \in[0,1]\}$, where $\lambda_{i}>0$ and $\mu_{i}>0$ are the constant transport velocities respectively associated to equations (1) and (2), and where the in-domain coupling terms $\sigma_{i}^{+}$and $\sigma_{i}^{-}$are continuous functions. The associated initial conditions are denoted $u_{i}^{0}(\cdot)=u_{i}(0, \cdot)$ and $v_{i}^{0}(\cdot)=v_{i}(0, \cdot)$ and are defined in $L^{2}([0,1], \mathbb{R})$. These subsystems are connected through their boundary conditions, which satisfy

$$
u_{i}(t, 0)=q_{i, i} v_{i}(t, 0)+q_{i-1, i} u_{i-1}(t, 1)+\delta_{1}^{i} V(t),
$$

and

$$
v_{i}(t, 1)=\rho_{i, i} u_{i}(t, 1)+\rho_{i, i+1} v_{i+1}(t, 0)+\delta_{n-1}^{i} C X(t)
$$

where the different coupling and $\rho_{i j}$ are constant and where $V$ is a real-valued control input. By convention $q_{0,1}=$ 0 and $\rho_{n-1, n}=0$. The notation $\delta_{j}^{i}$ stands for the Kronecker symbol (i.e. $\delta_{j}^{i}=1$ if $i=j$, and $\delta_{j}^{i}=0$ if $i \neq j$ ). The last subsystem is an ODE defined by

$$
\dot{X}(t)=A X(t)+B u_{n-1}(t, 1),
$$

where the matrices $A \in \mathbb{R}^{p \times p}, B \in \mathbb{R}^{p \times 1}, C \in \mathbb{R}^{1 \times p}$ are constant. The initial condition $X_{0}=X(0)$ belongs to $\mathbb{R}^{p}$. The well-posedness of system (1)-(5) is guaranteed by [9, Theorem A.6, page 254]. The system (1)-(5) is schematically pictured in Figure 1. The advantage of such a representation is that it highlights that the interaction between the different subsystems only occurs at the boundaries (black arrows). For instance, the output of the second subsystem entering the first subsystem could be seen as a kind of disturbance acting on the first subsystem (even if such a disturbance signal is indirectly modified by the corresponding output of the first subsystem). For a subsystem $i$, we will call the subsystem $i+1$ the downstream subsystem and $i-1$ the upstream subsystem.

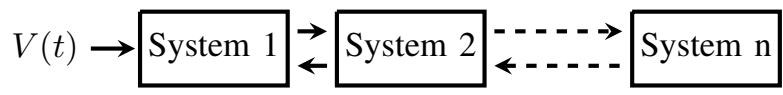

Fig. 1. Schematic representation of the system under consideration (1)-(5)

\section{B. Problem formulation}

The objective of this paper is to exponentially stabilize system (1)-(5) using the available control $V(t)$. The stabilization is done in the sense of the $\Xi$-norm defined by $\|(u, v, X)\|_{\Xi}=\left(\sum_{i=1}^{n-1}\left\|u_{i}\right\|_{L^{2}}^{2}+\left\|v_{i}\right\|_{L^{2}}^{2}+\|X\|_{\mathbb{R}^{p}}^{2}\right)^{\frac{1}{2}}$. This problem has already been solved in [4] for the case of a chain of PDEs (no ODE at the end of the chain). The method presented in [4] uses a triangular integral transformation to move most of the in-domain couplings at the actuated boundary. We believe that such an approach could be adjusted to the presence of the ODE. However, this would require deriving and solving a new set of complex kernel equations. Generally speaking, the approach proposed in [4] cannot be straightforwardly extended when adding new blocks inside the chain that have a different structure (i.e. adding ODEs in the chain or PDEs that have a different number of states). This explains why here, we develop a new recursive dynamics interconnection framework. Based on simple conditions placed on each independent subsystem, we derive a recursive control law that stabilizes the interconnected system (1)-(5). The main advantage of the proposed methodology is its potential extension to arbitrary chains of ODEs and PDEs. Moreover, the conditions derived in this paper are less restrictive than those used in [4].

\section{Description of the method}

The recursive dynamics interconnection framework we develop in this paper is based on a recursive approach. Considering the last subsystem as an independent system, we design a theoretical input that stabilizes it. This input is a state-feedback law that only depends on the last subsystem's state. Then, we consider the penultimate subsystem as an independent system only disturbed by the downstream subsystem's action. This penultimate subsystem acts on the last subsystem through its right output. For this system, we can design a (virtual) control input that stabilizes the system in the absence of the disturbance and, more importantly, that guarantees the convergence of its right output to the theoretical control law that stabilizes the last subsystem. Such a control law guarantees the stabilization of the last subsystem and finally of the penultimate subsystem. Due to the hyperbolic systems' transport structure, such a tracking control law may require a prediction of the downstream subsystem's state. Iterating such a procedure, it is possible to design a stabilizing control law $V(t)$ for the whole system.

One of the main advantages of such an approach is that, provided we can solve each subsystem's tracking problem, and correctly predict each subsystem's state (which is possible for ODEs and hyperbolic PDEs), it becomes straightforward to add new subsystems. This results in a simpler and more generic control design procedure than the one developed in [4]. 


\section{CONDITIONS TO DEVELOP A RECURSIVE DYNAMICS INTERCONNECTION FRAMEWORK}

In this section, we introduce several assumptions that are necessary to apply our new control framework. They correspond to a set of simple and natural conditions defined for each subsystem of (1)-(5). We show that these conditions are always satisfied for the class of systems we consider. In the next section, we will show that under these assumptions it becomes straightforward to stabilize the system (1)-(5). We start giving the following definition to qualify the interactions between the different subsystems.

Definition 1: For a subsystem $i<n$, we denote $\hat{V}_{i}(t)$ the action of the $(i-1)^{t h}$ subsystem on this subsystem. This function will be called virtual input acting on the $i^{\text {th }}$ subsystem. We also denote $\chi_{i}(t)$ the action of the $(i+1)^{t h}$ subsystem on the $i^{t h}$ subsystem. This function will be called virtual disturbance acting on the $i^{\text {th }}$ subsystem.

This terminology is justified by the recursive dynamics interconnection framework we develop since each subsystem is stabilized using its leftward neighbor. With these notations, we have for instance $\hat{V}_{2}(t)=q_{2,1} u_{1}(t, 1), \chi_{2}(t)=$ $\rho_{2,3} v_{3}(t, 0), \chi_{n-1}(t)=C X(t)$. For the last subsystem (ODE) we do not have any virtual output or virtual disturbance and we denote $\hat{V}_{n}(t)=u_{n-1}(t, 1)$. These different notations are summarized in Figure 2.

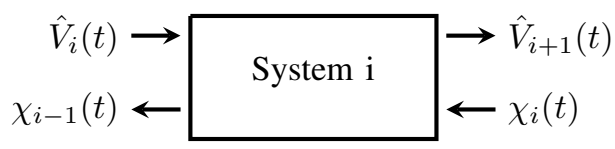

Fig. 2. Schematic representation of the interactions between the different subsystems

We are now ready to give the different assumptions for each subsystem and show that they are always satisfied.

\section{A. Delay-robustness assumption}

The first assumption we make aims to guarantee the existence of robustness margins for the closed-loop system.

Assumption 1: The open loop system (1)-(5) (i.e. $V(t) \equiv$ $0)$ in the absence of in-domain coupling terms (i.e. $\sigma_{i} \equiv 0$ ) and of the ODE (i.e $X \equiv 0$ ) is exponentially stable (in the sense of the $L^{2}$ norm).

It has been shown in [31] that a necessary condition to guarantee the existence of robustness margins for an arbitrary closed-loop system is that the open-loop transfer function must have a finite number of poles on the closed right halfplane. For a deeper discussion of the sensitivity wrt the delay parameter, the reader is referred to [32]. It is worth mentioning that for the system (1)-(5), [5], [8] proved that this implies that the open-loop system without in-domain couplings and without the ODE must be exponentially stable. Some explicit conditions to verify such an assumption can be found in [8] and [4]. Consequently, Assumption 1 is not restrictive as it is necessary for the existence of robustness margins for the closed-loop system.

\section{B. Stabilizability of each subsystem}

The second assumption we make guarantees the possibility to independently stabilize each subsystem.

Assumption 2: In the absence of the virtual disturbance $\chi_{i}$ (i.e. $\chi_{i}(t) \equiv 0$ ), the $i^{t h}$ subsystem subject to the virtual actuation $\hat{V}_{i}$ must be stabilizable by a state-feedback law. More precisely, if $i<n$ (resp. $i=n$ ), there exist a linear operator $\mathcal{K}_{i}:\left(L^{2}([0,1])\right)^{2} \rightarrow \mathbb{R}\left(\right.$ resp. $\left.\mathcal{K}_{n}: \mathbb{R}^{p} \rightarrow \mathbb{R}\right)$ such that $\hat{V}_{i}=\mathcal{K}_{i}\left(u_{i}, v_{i}\right)$ (resp. $\left.\hat{V}_{n}=\mathcal{K}_{n}(X)\right)$ stabilizes the system in the absence of the virtual disturbance.

This assumption is natural, since to stabilize our interconnected system for any initial condition, we need to have each subsystem independently stabilizable. For the ODE to be stabilizable, we must have the existence of a matrix $K$ such that $A+B K$ is Hurwitz. (i.e. has all its eigenvalues in the complex left half plane). Indeed, if $\hat{V}_{n}(t)=K X(t)$, then the ODE system is stabilized. Let us now consider the $i^{t h}$ subsystem $(i \in\{1,2, \cdots, n-1\})$ with the virtual actuation $\hat{V}_{i}(t)$. Assuming that $\chi_{i}(t) \equiv 0$, this PDE system rewrites

$$
\begin{aligned}
& \partial_{t} u_{i}(t, x)+\lambda_{i} \partial_{x} u_{i}(t, x)=\sigma_{i}^{+}(x) v_{i}(t, x) \\
& \partial_{t} v_{i}(t, x)-\mu_{i} \partial_{x} v_{i}(t, x)=\sigma_{i}^{-}(x) u_{i}(t, x),
\end{aligned}
$$

with the boundary conditions

$$
\begin{aligned}
& u_{i}(t, 0)=q_{i, i} v_{i}(t, 0)+\hat{V}_{i}(t), \\
& v_{i}(t, 1)=\rho_{i, i} u_{i}(t, 1) .
\end{aligned}
$$

Let us consider the following backstepping transformation adjusted from [6], [16], [42] and defined as

$$
\begin{aligned}
& \left(\begin{array}{c}
\alpha_{i}(t, x) \\
\beta_{i}(t, x)
\end{array}\right)=\left(\begin{array}{l}
u_{i}(t, x) \\
v_{i}(t, x)
\end{array}\right) \\
& -\int_{x}^{1}\left(\begin{array}{ll}
K_{i}^{u u}(x, y) & K_{i}^{u v}(x, y) \\
K_{i}^{v u}(x, y) & K_{i}^{v v}(x, y)
\end{array}\right)\left(\begin{array}{l}
u_{i}(t, y) \\
v_{i}(t, y)
\end{array}\right) d y,
\end{aligned}
$$

where the kernels $K_{i}$ are $L^{\infty}$ functions defined on the upper triangular part of the unit square $\mathcal{T}=\left\{(x, y) \in[0,1]^{2}, x \leq\right.$ $y\}$. They satisfy the following set of equations

$$
\begin{aligned}
& \lambda_{i} \partial_{x} K_{i}^{u u}(x, y)+\lambda_{i} \partial_{y} K_{i}^{u u}(x, y)=-\sigma_{i}^{-}(y) K_{i}^{u v}(x, y) \\
& \lambda_{i} \partial_{x} K_{i}^{u v}(x, y)-\mu_{i} \partial_{y} K_{i}^{u v}(x, y)=-\sigma_{i}^{+}(y) K_{i}^{u u}(x, y) \\
& \mu_{i} \partial_{x} K_{i}^{v u}(x, y)-\lambda_{i} \partial_{y} K^{v u}(x, y)=\sigma_{i}^{-}(y) K_{i}^{u v}(x, y), \\
& \mu_{i} \partial_{x} K_{i}^{v v}(x, y)+\mu_{i} \partial_{y} K^{v v}(x, y)=\sigma_{i}^{+}(y) K_{i}^{u v}(x, y), \\
& K_{i}^{u v}(x, x)=-\frac{\sigma_{i}^{+}}{\lambda_{i}+\mu_{i}}, K_{i}^{u v}(x, x)=\frac{\sigma_{i}^{-}}{\lambda_{i}+\mu_{i}} \\
& K_{i}^{u u}(x, 1)=\rho_{i, i} \frac{\mu_{i}}{\lambda_{i}} K_{i}^{u v}(x, 1), K^{v v}(x, 1)=0
\end{aligned}
$$

It has been proved in [16], [42] that this set of equations admits a unique solution. Defining $\hat{V}_{i}^{B S} \doteq \mathcal{K}_{i}\left(u_{i}(t, \cdot), v_{i}(t, \cdot)\right)$ (and therefore defining the operators $\mathcal{K}_{i}$ in Assumption 2) as

$$
\begin{aligned}
\hat{V}_{i}^{B S}(t)=-q_{i, i} v_{i}(t, 0) & +\int_{0}^{1} K_{i}^{u u}(0, y) u_{i}(t, y) d y \\
& +\int_{0}^{1} K_{i}^{u v}(0, y) v_{i}(t, y) d y,
\end{aligned}
$$

the transformation (10) maps the system (6)-(9) to the target system

$$
\begin{aligned}
& \partial_{t} \alpha_{i}(t, x)+\lambda_{i} \partial_{x} \alpha_{i}(t, x)=0, \\
& \partial_{t} \beta_{i}(t, x)-\mu_{i} \partial_{x} \beta_{i}(t, x)=\lambda_{i} K^{v u}(x, 1) \rho_{i} \alpha_{i}(t, 1),
\end{aligned}
$$

with the boundary conditions

$$
\alpha_{i}(t, 0)=0, \beta_{i}(t, 1)=\rho_{i, i} \alpha_{i}(t, 1) .
$$


This target system is exponentially stable. This in turns implies the exponential stability of the original system due to the invertibility of the transformation (10). However, it is worth mentioning that the control law $\hat{V}_{i}^{B S}(t)$ cancels the boundary reflection terms. As shown in [6], this may lead to vanishing delay margins. To avoid this problem and make the control law strictly proper, we can combine it with a well-tuned low pass filter as done in [7].

\section{Output tracking in presence of a disturbance signal}

The proposed approach requires each subsystem to be able to track any arbitrary function. More precisely, we have the following assumption

Assumption 3: Consider a subsystem $i<n$ and define $\Phi_{i} \in L_{\text {loc }}^{2}\left(\mathbb{R}^{+}\right)$an arbitrary known function. Let us assume that the virtual disturbance $\chi_{i}$ acting on this subsystem is known. Then, there exists a control law $\hat{V}_{i}$ that exponentially tracks the function $\hat{V}_{i+1}(t)$ to the desired function $\Phi_{i}(t)$. Moreover, if $\chi_{i}(t) \equiv \Phi_{i}(t) \equiv 0$, then, such a control law stabilizes the $i^{\text {th }}$ subsystem. More precisely, there exist linear causal operators $\mathcal{L}_{i}$, such that $\hat{V}_{i}(t)=\mathcal{K}_{i}\left(u_{i}(t, \cdot), v_{i}(t, \cdot)\right)+$ $\mathcal{L}_{i}\left(\Phi_{i}\left(t+\frac{1}{\lambda_{j}}\right), \chi_{i}\left(\left[t, t+\frac{1}{\lambda_{i}}\right]\right)\right)$, with the operators $\mathcal{K}_{i}$ being defined in Assumption 2 and (17), and the operators $\mathcal{L}_{i}$ defined later in this section.

The function $\hat{V}_{i+1}(t)$ (virtual input of the $(i+1)^{t h}$ subsystem) corresponds to the right output of the subsystem $i$. The fact that the operators $\mathcal{L}_{i}$ require future values of the $\Phi_{i}$ and $\chi_{i}$ is related to the transport delay to go from the left boundary of the PDE (where is the actuation) to the right boundary (where is defined the output we want to track). This assumption does not have to be fulfilled for the last subsystem (i.e. for the ODE), as explained in Section II-C. Since the function $\hat{V}_{i+1}$ is defined by $\hat{V}_{i+1}(t)=q_{i, i+1} u_{i}(t, 1)$ (with the convention $\left.q_{n-1, n}=1\right)$, we are led to the following condition: for all $1<i \leq n$, we have $q_{i-1, i} \neq 0$. Indeed, if it is not the case, we cannot track any arbitrary function since the output will always be zero. Regarding our class of PDEs subsystems we can adjust the approach presented in [26] to solve this tracking problem. More precisely, in the presence of a disturbance $\chi_{i}(t)$ acting on the $i^{t h}$ system, the transformation (10) maps the system (6)-(9) to the target system

$$
\begin{aligned}
& \partial_{t} \alpha_{i}+\lambda_{i} \partial_{x} \alpha_{i}=-\mu_{i} K_{i}^{u v}(x, 1) \chi_{i}(t) \\
& \partial_{t} \beta_{i}-\mu_{i} \partial_{x} \beta_{i}=\lambda_{i} K_{i}^{v u}(x, 1) \alpha_{i}(t, 1),
\end{aligned}
$$

with the boundary conditions

$$
\begin{aligned}
\alpha_{i}(t, 0) & =V_{i}^{t r}(t), \\
\beta_{i}(t, 1) & =\rho_{i, i} \alpha_{i}(t, 1)+\chi_{i}(t) .
\end{aligned}
$$

where we have defined $V_{i}^{t r}(t)=\hat{V}_{i}(t)-V_{i}^{B S}(t)$. The backstepping transformation (10) does not modify the function $u_{i}(t, 1)$. Indeed, we have $\hat{V}_{i+1}(t)=q_{i+1, i} u_{i}(t, 1)=$ $q_{i+1, i} \alpha_{i}(t, 1)$ (with the convention $q_{n, n-1}=1$ ). Applying the method of characteristics on equation (21), we have

$$
\begin{aligned}
\alpha_{i}(t, 1) & =V_{i}^{t r}\left(t-\frac{1}{\lambda_{i}}\right) \\
& -\mu_{i} \int_{0}^{\frac{1}{\lambda_{i}}} K_{i}^{u v}\left(x-\lambda_{i} s, 1\right) \chi_{i}(t-s) d s .
\end{aligned}
$$

Thus, we can simply choose $\hat{V}_{i}^{t r} \doteq \mathcal{L}_{i}\left(\Phi_{i}\left(t+\frac{1}{\lambda_{i}}\right), \chi_{i}([t, t+\right.$ $\left.\left.\frac{1}{\lambda_{i}}\right]\right)$ ) (fixing the operators $\mathcal{L}_{i}$ in Assumption 3) as

$$
\begin{aligned}
\hat{V}_{i}^{t r}(t) & =\frac{1}{q_{i+1, i}} \Phi_{i}\left(t+\frac{1}{\lambda_{i}}\right) \\
& +\mu_{i} \int_{0}^{\frac{1}{\lambda_{i}}} K_{i}^{u v}\left(1-\lambda_{i} s, 1\right) \chi_{i}\left(t+\frac{1}{\lambda_{i}}-s\right) d s,
\end{aligned}
$$

to guarantee the convergence of $y_{i}(t)=u_{i}(t, 1)=\alpha_{i}(t, 1)$ to $\Phi_{i}(t)$. If the functions $\Phi_{i}$ and $\chi_{i}$ converge to zero, so does the state $\alpha_{i}(t, 1)$ and so does the whole $i^{t h}$-subsystem due to its transport structure.

\section{Prediction of the state}

The tracking operators $\mathcal{L}_{i}$ require future values of the functions $\Phi_{i}$ and $\chi_{i}$. Since these functions depend on the states of the downstream subsystems, we must be able to predict future values of these states. This leads us to the following assumption.

Assumption 4: For all $x \in[0,1]$, it is possible to obtain a $\sum_{j=1}^{i-1} \frac{1}{\lambda_{j}}+\frac{x}{\lambda_{i}}$ units of time ahead prediction of the PDE states $u_{i}(t, x)$ and $v_{i}(t, x)$, and a $\sum_{j=1}^{n-1} \frac{1}{\lambda_{j}}+\frac{x}{\lambda_{i}}$ units of time ahead prediction of the ODE $X$. More precisely there exist predictor functions $P_{v_{i}}, P_{u_{i}}$ and $P_{X}$ such that for all $t>0, P_{v_{i}}(t, x)=v_{i}\left(t+\sum_{j=1}^{i-1} \frac{1}{\lambda_{j}}+\frac{x}{\lambda_{i}}, x\right), P_{u_{i}}(t, x)=$ $u_{i}\left(t+\sum_{j=1}^{i-1} \frac{1}{\lambda_{j}}+\frac{x}{\lambda_{i}}, x\right), P_{X}(t)=X\left(t+\sum_{j=1}^{n-1} \frac{1}{\lambda_{j}}\right)$.

Let us explicitly design these predictors. In what follows, to avoid useless case disjunctions, we use the convention $\rho_{n-1, n}=C$ and $v_{n}(t, 0)=X$. Applying the transformation (10) to each subsystem (6)-(9), we obtain the target system

$$
\begin{gathered}
\partial_{t} \alpha_{i}(t, x)+\lambda_{i} \partial_{x} \alpha_{i}(t, x)=f_{i}^{+}(x) v_{i+1}(t, 0) \\
\partial_{t} \beta_{i}(t, x)-\mu_{i} \partial_{x} \beta_{i}(t, x)=f_{i}^{-}(x) u_{i}(t, 1),
\end{gathered}
$$

with the boundary conditions

$$
\begin{aligned}
& \alpha_{i}(t, 0)=q_{i, i} v_{i}(t, 0)+q_{i, i-1} u_{i-1}(t, 1)+\delta_{1}^{i} V(t) \\
& +\int_{0}^{1} L_{i}^{\alpha \alpha} \alpha_{i}(t, \xi)+L_{i}^{\alpha \beta} \beta_{i}(t, \xi) \\
& \beta_{i}(t, 1)=\rho_{i, i} \alpha_{i}(t, 1)+\rho_{i, i+1} v_{i+1}(t, 0)+\delta_{n-1}^{i} C X .
\end{aligned}
$$

where we recall that the kernels $L_{i}$ are the inverse kernels of the kernels $K_{i}^{*}$ (see [16], [42] for details), and where we have defined $f_{i}^{-}(x)=\lambda_{i} K_{i}^{v u}(x, 1)$ and $f_{i}^{+}(x)=$ $-\mu_{i} \rho_{i, i+1} K_{i}^{u v}(x, 1)$. With these definitions, equations (27)(28) correspond to the tracking system (21)-(22). To these PDEs, we add the ODE (5). Note that we decided to preserve the terms $u_{i}(t, 1)$ and $v_{i}(t, 0)$ inside the target systems to avoid adding integral terms in $\alpha$ and $\beta$. We will first design a predictor for the states $u_{i}(t, 1), v_{i}(t, 0), \alpha_{i}(t, 0)$ and $X(t)$ and show that these predictors give us the wanted predictor for the whole original states. Using the method of characteristics, we have for $i<n+1$

$$
\begin{aligned}
u_{i}(t, 1)= & \alpha_{i}\left(t-\frac{1}{\lambda_{i}}, 0\right) \\
& +\int_{0}^{\frac{1}{\lambda_{i}}} f_{i}^{+}\left(1-\nu \lambda_{i}\right) v_{i+1}(t-\nu, 0) d \nu,
\end{aligned}
$$

(for $i=n-1$, the term $v_{i+1}(t, 0)$ has to be replaced by $X(t)$ ). Following the approach given in [8] (we choose to 
skip the complete proof here, due to space restrictions), we obtain

$v_{i}(t, 0)=\rho_{i, i} u_{i}\left(t-\frac{1}{\mu_{i}}, 1\right)+\rho_{i, i+1} v_{i+1}\left(t-\frac{1}{\mu_{i}}, 0\right)+\int_{0}^{\tau_{i}} g_{i}^{1}(\nu)$

$\alpha_{i}(t-\nu, 0)+g_{i}^{2}(\nu) u_{i}(t-\nu, 1)+g_{i}^{3}(\nu) v_{i+1}(t-\nu, 0) d \nu$.

where $\tau_{i}=\frac{1}{\lambda_{i}}+\frac{1}{\mu_{i}}$ and where the functions $g_{i}^{1}, g_{i}^{2}$ and $g_{i}^{3}$ are defined by

$$
\begin{aligned}
& g_{i}^{1}(\nu)=-\mathbb{1}_{\left[0, \frac{1}{\lambda_{i}}\right]}(\nu) \lambda_{i} L_{i}^{\beta \alpha}\left(0, \lambda_{i} \nu\right), \\
& g_{i}^{2}(\nu)=\mathbb{1}_{\left[0, \frac{1}{\mu_{i}}\right]}(\nu)\left(f_{i}^{-}\left(\mu_{i} \nu\right)-\mu_{i} \rho_{i, i} L_{i}^{\beta \beta}\left(0,1-\mu_{i} \nu\right)\right. \\
& \left.-\int_{0}^{1-\mu_{i} \nu} L_{i}^{\beta \beta}(0, \xi) f_{i}^{-}\left(\xi+\mu_{i} \nu\right) d \xi\right) \\
& g_{i}^{3}(\nu)=-\mathbb{1}_{\left[0, \frac{1}{\mu_{i}}\right]}(\nu)\left(\mu_{i} \rho_{i, i+1} L_{i}^{\beta \beta}\left(0,1-\mu_{i} \nu\right)\right) \\
& -\mathbb{1}_{\left[0, \frac{1}{\lambda_{i}}\right]}(\nu)\left(\int_{\lambda_{i} \nu}^{1} L_{i}^{\beta \alpha}(0, \xi) f_{i}^{+}\left(\xi-\lambda_{i} \nu\right) d \xi\right)
\end{aligned}
$$

where $\mathbb{1}_{\Omega}$ denotes the characteristic function of the set $\Omega$. Similarly, we obtain

$$
\begin{aligned}
& \alpha_{i}(t, 0)=q_{i, i} v_{i}(t, 0)+q_{i, i-1} u_{i-1}(t, 1)+\delta_{i}^{1} V(t) \\
& +\int_{0}^{\tau_{i}} k_{i}^{1}(\nu) \alpha_{i}(t-\nu, 0)+k_{i}^{2}(\nu) u_{i}(t-\nu, 1) d \nu \\
& +\int_{0}^{\tau_{i}} k_{i}^{3}(\nu) v_{i+1}(t-\nu, 0) d \nu .
\end{aligned}
$$

where the functions $k_{i}^{1}, k_{i}^{2}$ and $k_{i}^{3}$ are defined by

$$
\begin{aligned}
& k_{i}^{1}(\nu)=\mathbb{1}_{\left[0, \frac{1}{\lambda_{i}}\right]}(\nu) \lambda_{i} L_{i}^{\alpha \alpha}\left(0, \lambda_{i} \nu\right), \\
& k_{i}^{2}(\nu)=\mathbb{1}_{\left[0, \frac{1}{\mu_{i}}\right]}(\nu)\left(\mu_{i} \rho_{i, i} L_{i}^{\alpha \beta}\left(0,1-\mu_{i} \nu\right)\right. \\
& \left.+\int_{0}^{1-\mu_{i} \nu} L_{i}^{\alpha \beta}(0, \xi) f_{i}^{-}\left(\xi+\mu_{i} \nu\right) d \xi\right) \\
& k_{i}^{3}(\nu)=\mathbb{1}_{\left[0, \frac{1}{\mu_{i}}\right]}(\nu)\left(\mu_{i} \rho_{i, i+1} L_{i}^{\alpha \beta}\left(0,1-\mu_{i} \nu\right)\right) \\
& +\mathbb{1}_{\left[0, \frac{1}{\lambda_{i}}\right]}(\nu)\left(\int_{\lambda_{i} \nu}^{1} L_{i}^{\alpha \alpha}(0, \xi) f_{i}^{+}\left(\xi-\lambda_{i} \nu\right) d \xi\right) .
\end{aligned}
$$

We can now define the state predictors $P_{\alpha_{i}}(t, s), P_{v_{i}}(t, s)$, $P_{v_{i}}(t, s)$ and $P_{X}(t, s)$ (see [11], [14]). Let us define $\tau=$ $\sum_{i} \tau_{i}$ as the total transport delay. Inspired by [7], let us define for $t \geq 0$ and $s \in\left[t-\tau-\sum_{j=1}^{i-1} \frac{1}{\lambda_{j}}, t\right], P_{\alpha_{i}}(t, s)$ (resp. $P_{v_{i}}(t, s)$ ) as the state prediction of $\alpha_{i}(t, 0)$, (resp. $v_{i}(t, 0)$ ), ahead a time $\sum_{j=1}^{i-1} \frac{1}{\lambda_{j}}$, for $s \in\left[t-\tau-\sum_{j=1}^{i} \frac{1}{\lambda_{j}}, t\right], P_{u_{i}}(t, s)$ as the state prediction of $u_{i}(t, 1)$, ahead a time $\sum_{j=1}^{i} \frac{1}{\lambda_{j}}$, and for $s \in\left[t-\tau-\sum_{j=1}^{n-1} \frac{1}{\lambda_{j}}, t\right], P_{X}(t, s)$ as the state prediction of $X(t)$, ahead a time $\sum_{j=1}^{n-1} \frac{1}{\lambda_{j}}$. They are defined by the set of equations (40)-(43). From these definitions, we immediately have

$$
\begin{aligned}
& P_{\alpha_{i}}(t, s)=\alpha_{i}\left(s+\sum_{j=1}^{i-1} \frac{1}{\lambda_{j}}, 0\right), s \in\left[t-\tau-\sum_{j=1}^{i-1} \frac{1}{\lambda_{j}}, t\right], \\
& P_{v_{i}}(t, s)=v_{i}\left(s+\sum_{j=1}^{i-1} \frac{1}{\lambda_{j}}, 0\right), s \in\left[t-\tau-\sum_{j=1}^{i-1} \frac{1}{\lambda_{j}}, t\right],
\end{aligned}
$$

$$
\begin{aligned}
& P_{u_{i}}(t, s)=u_{i}\left(s+\sum_{j=1}^{i} \frac{1}{\lambda_{j}}, 1\right), s \in\left[t-\tau-\sum_{j=1}^{i} \frac{1}{\lambda_{j}}, t\right], \\
& P_{X}(t, s)=X\left(s+\sum_{j=1}^{n-1} \frac{1}{\lambda_{j}}\right), s \in\left[t-\tau-\sum_{j=1}^{n-1} \frac{1}{\lambda_{j}}, t\right] .
\end{aligned}
$$

From these predictions, it is straightforward to obtain the predictions of the states $\alpha_{i}(t, x)$ and $\beta_{i}(t, x)$, using the fact that $\alpha_{i}(t, x)=\alpha_{i}\left(t-\frac{x}{\lambda_{i}}, 0\right)+\int_{0}^{\frac{x}{\lambda_{i}}} f_{i}^{+}\left(x-\lambda_{i} \nu\right) v_{i+1}(t-$ $\nu, 0) d \nu$, and

$$
\begin{gathered}
\beta_{i}(t, x)=\rho_{i, i} u_{i}\left(t-\frac{1-x}{\mu_{i}}, 1\right)+\rho_{i, i+1} v_{i+1}\left(t-\frac{1-x}{\mu_{i}}, 0\right) \\
+\int_{0}^{\frac{1-x}{\mu_{i}}} f_{i}^{-}\left(x+\mu_{i} \nu\right) u_{i}(t-\nu, 1) d \nu .
\end{gathered}
$$

Finally, using the invertibility of the backstepping transformation (10), we obtain the desired predictions for the original PDE and ODE states.

\section{STATE FeEdback CONTROL LAW}

We now have all the tools to apply our recursive dynamics interconnection framework.

Theorem 1: For $i \in[1, n-1]$, let us define the following sequences

$$
\begin{aligned}
\hat{V}_{n}(t) & =\mathcal{K}_{n}\left(P_{X}\left(t-\sum_{j=1}^{n-1} \frac{1}{\lambda_{j}}\right)\right), \\
\chi_{n-1}(t) & =C P_{X}\left(t-\sum_{j=1}^{n-1} \frac{1}{\lambda_{j}}\right), \\
\hat{V}_{i}(t) & =\mathcal{K}_{i}\left(P_{u_{i}}\left(t-\sum_{j=1}^{i-1} \frac{1}{\lambda_{j}}-\frac{x}{\lambda_{i}}, x\right), P_{v_{i}}\left(t-\sum_{j=1}^{i-1} \frac{1}{\lambda_{j}}\right.\right. \\
& \left.\left.-\frac{x}{\lambda_{i}}, x\right)\right)+\mathcal{L}_{i}\left(\hat{V}_{i+1}\left(t+\frac{1}{\lambda_{i}}\right), \chi_{i}\left(t+\frac{1}{\lambda_{i}}\right)\right), \\
\chi_{i-1}(t) & =\rho_{i-1, i} P_{v_{i}}\left(t-\sum_{j=1}^{i-1} \frac{1}{\lambda_{j}}, 0\right), i>1,
\end{aligned}
$$

where the operators $\mathcal{K}_{i}$ are defined in Assumption 2, the operators $\mathcal{L}_{i}$ are defined in Assumption 3 and the predictors $P_{u_{i}}, P_{v_{i}}$ and $P_{X}$ are defined in Assumption 4. Then, the control law $V(t)=\hat{V}_{1}(t)$ exponentially stabilizes the system (1)-(5) in the sense of the $\Xi$-norm.

Proof: The control law $V(t)$ is well-defined and causal, due to the definition of the different predictors (Assumption 4.) Then, applying Assumption 3 on each subsystem, we obtain that $u_{n-1}(t, 1)$ exponentially converges to $\mathcal{K}_{n}(X(t))$. Consequently, $X(t)$ exponentially converges to zero. Using Assumption 3, we can recursively show that each subsystem exponentially converges to zero starting from $i=(n-1)$. This concludes the proof.

One major advantage of the proposed framework and of the recursive design proposed in Theorem 1 is that it can easily be extended to different classes of subsystems (ODEs, non-scalar PDEs), as long as Assumptions 1, 2, 3, 4 can be verified. One must be aware that Theorem 1 completely neglects the robustness aspects of the system. More precisely, the operators $\mathcal{K}$ we have designed in Section 2 cancel all the boundary reflection terms and may not be strictly proper 


$$
\begin{aligned}
& P_{\alpha_{i}}(t, s)=\left\{\begin{array}{l}
\alpha_{i}\left(s+\sum_{j=1}^{i-1} \frac{1}{\lambda_{j}}, 0\right) \quad \text { if } s \in\left[t-\tau-\sum_{j=1}^{i-1} \frac{1}{\lambda_{j}}, t-\sum_{j=1}^{i-1} \frac{1}{\lambda_{j}}\right] \\
q_{i, i} P_{v_{i}}(t, s)+q_{i, i-1} P_{u_{i-1}}(t, s)+\delta_{i}^{1} V(s)+\int_{0}^{\tau_{i}} k_{i}^{1}(\nu) P_{\alpha_{i}}(t, s-\nu)+k_{i}^{2}(\nu) P_{u_{i}}\left(t, s-\nu-\frac{1}{\lambda_{i}}\right)+d \nu \\
+\int_{0}^{\tau_{i}} k_{i}^{3}(\nu) P_{v_{i+1}}\left(t, s-\nu-\frac{1}{\lambda_{i}}\right) d \nu \text { otherwise, }
\end{array}\right. \\
& P_{v_{i}}(t, s)=\left\{\begin{array}{l}
v_{i}\left(s+\sum_{j=1}^{i-1} \frac{1}{\lambda_{j}}, 0\right) \text { if } s \in\left[t-\tau-\sum_{j=1}^{i-1} \frac{1}{\lambda_{j}}, t-\sum_{j=1}^{i-1} \frac{1}{\lambda_{j}}\right] \\
\rho_{i, i+1} P_{v_{i+1}}\left(t, s-\frac{1}{\lambda_{i}}-\frac{1}{\mu_{i}}\right)+\rho_{i, i} P_{u_{i}}\left(t, s-\frac{1}{\lambda_{i}}-\frac{1}{\mu_{i}}\right)+\int_{0}^{\tau_{i}} g_{i}^{1}(\nu) P_{\alpha_{i}}(t, s-\nu)+g_{i}^{2}(\nu) P_{u_{i}}\left(t, s-\nu-\frac{1}{\lambda_{i}}\right) d \nu \\
+\int_{0}^{\tau_{i}} g_{i}^{3}(\nu) P_{v_{i+1}}\left(t, s-\nu-\frac{1}{\lambda_{i}}\right) d \nu \text { otherwise, }
\end{array}\right. \\
& P_{u_{i}}(t, s)=\left\{\begin{array}{l}
u_{i}\left(s+\sum_{j=1}^{i} \frac{1}{\lambda_{j}}, 0\right) \text { if } s \in\left[t-\tau-\sum_{j=1}^{i} \frac{1}{\lambda_{j}}, t-\sum_{j=1}^{i} \frac{1}{\lambda_{j}}\right] \\
P_{\alpha_{i}}(t, s)+\int_{0}^{\frac{1}{\lambda_{i}}} f_{i}^{+}\left(1-\nu \lambda_{i}\right) P_{v_{i+1}}(t, s-\nu) d \nu \text { otherwise, }
\end{array}\right. \\
& P_{X}(t, s)=\left\{\begin{array}{l}
X\left(s+\sum_{j=1}^{n-1} \frac{1}{\lambda_{j}}\right) \quad \text { if } s \in\left[t-\tau-\sum_{j=1}^{n-1} \frac{1}{\lambda_{j}}, t-\sum_{j=1}^{n-1} \frac{1}{\lambda_{j}}\right] \\
\mathrm{e}^{A \sum_{j=1}^{n-1} \frac{1}{\lambda_{j}}}\left(X(s)+\int_{s}^{s+\sum_{j=1}^{n-1} \frac{1}{\lambda_{j}}} \mathrm{e}^{A(s-\nu)} B P_{u_{n-1}}\left(t, \nu-\sum_{j=1}^{n-1} \frac{1}{\lambda_{j}}\right) d \nu\right) \text { otherwise. }
\end{array}\right.
\end{aligned}
$$

(that may lead to zero robustness margins as shown in [6]). However, due to Assumption 1, it is possible to low-pass filter these operators and make them strictly proper, as done in [7].

\section{Applications}

\section{A. Simulation results}

The proposed control law has been tested in simulations using Matlab. The PDE systems are simulated using a classical finite volume method based on a Godunov scheme [30]. We used 61 spatial discretization points (and a CFL number of 1). The predictors are implemented using a backward Euler approximation of the integral terms. The numerical values used are: $n=3, \lambda_{1}=1, \lambda_{2}=2, \mu_{1}=1.3, \mu_{2}=1.8$, $\sigma_{1}^{+}=1, \sigma_{1}^{-}=0.4, \sigma_{2}^{+}=-0.3, \sigma_{2}^{-}=0.7, q_{11}=0.5, q_{12}=$ $0.3, q_{22}=0.2, \rho_{11}=0.275, \rho_{12}=0.4, \rho_{22}=0.4, A=$ $0.1, B=0.1, C=0.1$ These coefficients are chosen such that the whole system is unstable in open-loop. We have pictured in Figure 3 the evolution of the $\Xi$-norm of the system in open-loop and in closed-loop. As expected, the proposed control law guarantees the exponential stability. Note that the control law has been filtered by a low-pass filter to guarantee the existence of robustness margins. The important amplitude of the norm of the closed-loop system during the transient is due to the important initial value of the ODE (compared to the values of the PDEs states). Compensating this state implies propagating it through the different PDEs, which may create an artificial high norm during the transient.

\section{B. Illustrative example}

Since the control law resulting from our recursive dynamics framework requires the knowledge of all the state, it is yet unusable for practical applications. However, combined with

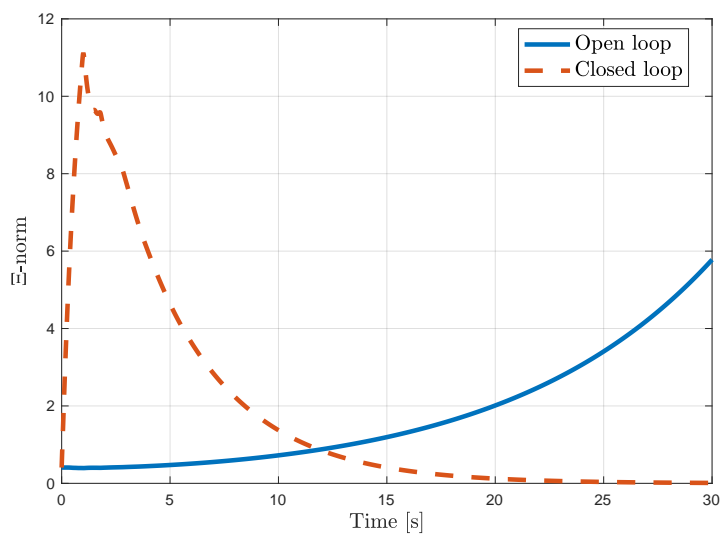

Fig. 3. Evolution of the $\Xi$-norm of the open-loop system (1)-(5) and of the closed-loop system using the control law defined in Theorem 1

a state observer, it could be applied to industrial problems. More precisely, using the mathematical model proposed by [1], [21], our framework could help controlling drilling devices.

\section{CONCLUDING REMARKS}

In this paper, we have designed a full-state feedback control law that stabilizes a network of interconnected scalar linear hyperbolic systems coupled with an ODE at the end of the chain. We propose a recursive dynamics interconnection framework that consists of solving simpler tracking and stateprediction problems for each subsystem. Then, it becomes possible to design a stabilizing control law for the whole system recursively. The proposed control law can be lowpass filtered in order to guarantee the existence of robustness 
margins. We believe that this new approach can be easily adapted to stabilize more general classes of interconnected systems due to its relative simplicity (as opposed to starting a new design with an ad hoc transformation). In future works, we will consider the design of a state-observer. It is a crucial step to obtain an output-feedback law. Such an observer should encompass the prediction of the state. We will also consider a more general class of networks, for which we may have non-scalar PDE subsystems or for which we have ODE subsystems between two PDE subsystems.

\section{REFERENCES}

[1] U. J. F. Aarsnes and O. M. Aamo. Linear stability analysis of selfexcited vibrations in drilling using an infinite dimensional model. Journal of Sound and Vibration, 360:239-259, jan 2016.

[2] U.J.F Aarsnes, R. Vazquez, F. Di Meglio, and M. Krstic. Delay robust control design of under-actuated PDE-ODE-PDE systems. In American and Control Conference, 2019.

[3] A. Araujo, A. Carvalho, and J. L. Martins de Carvalho. A modular approach for modeling and simulation of semiconductor power devices. In IECON '98. Proceedings of the 24th Annual Conference of the IEEE Industrial Electronics Society (Cat. No.98CH36200), volume 1, pages 331-335 vol.1, 1998.

[4] J. Auriol. Output feedback stabilization of an underactuated cascade network of interconnected linear pde systems using a backstepping approach. Automatica, 117:108964, 2020.

[5] J. Auriol and F. Bribiesca-Argomedo. Delay-robust stabilization of an $n+m$ hyperbolic pde-ode system. In IEEE Conference on Decision and Control and European Control Conference, 2019.

[6] J. Auriol, F. Bribiesca Argomedo, D. Bou Saba, M. Di Loreto, and F. Di Meglio. Delay-robust stabilization of a hyperbolic PDE-ODE system. Automatica, 95:494-502, 2018

[7] J. Auriol, F. Bribiesca-Argomedo, and D. Bresch-Pietri. Stabilization of an underactuated $1+2$ linear hyperbolic system with a proper control. In Conference on Decision and Control, 2020.

[8] J. Auriol and F. Di Meglio. An explicit mapping from linear first order hyperbolic PDEs to difference systems. Systems \& Control Letters, 123:144-150, 2019.

[9] G. Bastin and J.-M. Coron. Stability and boundary stabilization of 1-D hyperbolic systems. Springer, 2016.

[10] G. Bastin, J.-M. Coron, and S. O. Tamasoiu. Stability of linear densityflow hyperbolic systems under PI boundary control. Automatica, 53:37-42, 2015.

[11] N. Bekiaris-Liberis. Simultaneous compensation of input and state delays for nonlinear systems. Systems and Control Letters, 73:96$102,2014$.

[12] N. Bekiaris-Liberis and M. Krstic. Nonlinear control under nonconstant delays. SIAM, 2013.

[13] D. Bou Saba, F. Bribiesca-Argomedo, M. Di Loreto, and D. Eberard. Strictly proper control design for the stabilization of $2 \times 2$ linear hyperbolic ODE-PDE-ODE systems. In Proceedings of the 58th IEEE Conference on Decision and Control, Nice, France, December 2019.

[14] D. Bresch-Pietri and F. Di Meglio. Prediction-based control of linear input-delay system subject to state-dependent state delay-application to suppression of mechanical vibrations in drilling. Proc. of the 2nd IFAC Workshop on Control of Systems Governed by Partial Differential Equations, 49(8):111-117, 2016.

[15] M. Burkhardt, H. Yu, and M. Krstic. Stop-and-go suppression in twoclass congested traffic. arXiv preprint arXiv:1905.06476, 2019.

[16] J-M Coron, R. Vazquez, M. Krstic, and G. Bastin. Local exponential $H^{2}$ stabilization of a $2 \times 2$ quasilinear hyperbolic system using backstepping. SIAM Journal on Control and Optimization, 51(3):20052035, 2013.

[17] G. A. de Andrade, R. Vazquez, and D. J Pagano. Backstepping stabilization of a linearized ODE-PDE rijke tube model. Automatica, 96:98-109, oct 2018.

[18] J. Deutscher, N. Gehring, and R. Kern. Output feedback control of general linear heterodirectional hyperbolic ode-pde-ode systems. Automatica, 95:472-480, 2018.

[19] N. Espitia, A. Girard, N. Marchand, and C. Prieur. Fluid-flow modeling and stability analysis of communication networks. IFACPapersOnLine, 50(1):4534-4539, 2017.

[20] N. Gehring. A systematic design of backstepping-based state feedback controllers for ode-pde-ode systems.

[21] C. Germay, V. Denoël, and E. Detournay. Multiple mode analysis of the self-excited vibrations of rotary drilling systems. Journal of Sound and Vibration, 325(1-2):362-381, aug 2009.

[22] Q. Gu and T. Li. Exact boundary controllability of nodal profile for quasilinear hyperbolic systems in a tree-like network. Mathematical Methods in the Applied Sciences, 34(8):911-928, 2011.
[23] Q. Gu and T. Li. Exact boundary controllability of nodal profile for unsteady flows on a tree-like network of open canals. Journal de Mathématiques Pures et Appliquées, 99(1):86-105, 2013.

[24] A Hayat. PI controller for the general saint-venant equations. 2019.

[25] A. Hayat and P. Shang. Exponential stability of density-velocity systems with boundary conditions and source term for the $h^{2}$ norm. 2019.

[26] L. Hu, F. Di Meglio, R. Vazquez, and M. Krstic. Control of homodirectional and general heterodirectional linear coupled hyperbolic PDEs. IEEE Transactions on Automatic Control, 61(11):3301-3314, 2016.

[27] A. Irscheid, N. Gehring, and J. Rudolph. Trajectory tracking control for a class of $2 \times 2$ hyperbolic pde-ode systems.

[28] M. Krstic and A. Smyshlyaev. Backstepping boundary control for first-order hyperbolic PDEs and application to systems with actuator and sensor delays. Systems \& Control Letters, 57(9):750-758, 2008.

[29] J. Ladiges, A. Fay, T. Holm, U. Hempen, L. Urbas, M. Obst, and T. Albers. Integration of modular process units into process control systems. IEEE Transactions on Industry Applications, 54(2):1870$1880,2018$.

[30] R. J. LeVeque. Finite volume methods for hyperbolic problems. Cambridge university press, 2002.

[31] H. Logemann, R. Rebarber, and G. Weiss. Conditions for robustness and nonrobustness of the stability of feedback systems with respect to small delays in the feedback loop. SIAM Journal on Control and Optimization, 34(2):572-600, 1996.

[32] W. Michiels and S.-I. Niculescu. Stability and stabilization of timedelay systems: an eigenvalue-based approach. SIAM, 2007.

[33] K. Mokhtari Jadid. Performance evaluation of virtual flow metering models and its application to metering backup and production allocation. 2017.

[34] N. Petit and P. Rouchon. Flatness of heavy chain systems. Proceedings of the 41st IEEE Conference on Decision and Control, 2002., 1(2):362-367, 2001.

[35] O.O Rodriguez-Diaz, D. Novella-Rodriguez, E. Witrant, and E. Franco-Mejia. Control strategies for ventilation networks in smallscale mines using an experimental benchmark. Asian Journal of Control, pages 129-166, 2020

[36] B. Saldivar, S. Mondié, S.-I. Niculescu, H. Mounier, and I. Boussaada. A control oriented guided tour in oilwell drilling vibration modeling. Annual Reviews in Control, 42:100 - 113, 2016.

[37] C. Schmuck, F. Woittennek, A. Gensior, and J. Rudolph. Feed-forward control of an HVDC power transmission network. IEEE Transactions on Control Systems Technology, 22(2):597-606, 2014.

[38] R. Simon, R. Kania, R. Zuckermann, V. Huebner, D. Jewell, S. Banville, S. Ng, L. Wang, S. Rosenberg, C. Marlowe, D. Spellmeyer, R. Tan, A. Frankel, D. Santi, F. Cohen, and P. Bartlett. Peptoids: A modular approach to drug discovery. Proc. Natl. Acad. Sci. U. S. A., 89:9367-9371, 101992.

[39] T. Strecker and O. M. Aamo. Output feedback boundary control of series interconnections of $2 \times 2$ semilinear hyperbolic systems. IFACPapersOnLine, 50(1):663-670, 2017.

[40] L. Su, W. Guo, J.-M. Wang, and M. Krstic. Boundary stabilization of wave equation with velocity recirculation. IEEE Transactions on Automatic Control, 62(9):4760-4767, 2017.

[41] A. Sunder, R. Mülhaupt, R. Haag, and H. Frey. Hyperbranched polyether polyols: A modular approach to complex polymer architectures. Advanced Materials, 12:235 - 239, 022000.

[42] R. Vazquez, J.-M. Coron, M. Krstic, and G. Bastin. Local exponential $H^{2}$ stabilization of a $2 \times 2$ quasilinear hyperbolic system using backstepping. In Decision and Control and European Control Conference (CDC-ECC), 2011 50th IEEE Conference on, pages 1329-1334. IEEE, 2011.

[43] J. Wang, M. Krstic, and Y. Pi. Control of a $2 \times 2$ coupled linear hyperbolic system sandwiched between 2 odes. International Journal of Robust and Nonlinear Control, 28(13):3987-4016, 2018.

[44] K. Wang and Q. Gu. Exact boundary controllability of nodal profile for quasilinear wave equations in a planar tree-like network of strings Mathematical Methods in the Applied Sciences, 37(8):1206-1218, 2014.

[45] H.-N. Wu and J.-W. Wang. Static output feedback control via pde boundary and ode measurements in linear cascaded ode-beam systems. Automatica, 50(11):2787-2798, 2014.

[46] H. Yu, J. Auriol, and M. Krstic. Simultaneous stabilization of traffic flow on two connected roads. IEEE American Control Conference, 2020. 